\title{
Chapter 11 \\ Autonomous Surgical Robotics at Task and Subtask Levels
}

\author{
Tamás Dániel Nagy \\ (iD) https://orcid.org/0000-0002-5485-9588 \\ Óbuda University, Hungary \\ Tamás Haidegger \\ iD https://orcid.org/0000-0003-1402-1139 \\ Óbuda University, Hungary
}

\section{ABSTRACT}

The revolution of minimally invasive procedures had a significant influence on surgical practice, opening the way to laparoscopic surgery, then evolving into robotics surgery. Teleoperated master-slave robots, such as the da Vinci Surgical System, has become a standard of care during the lastfew decades, performing over a million procedures per year worldwide. Many believe that the next big step in the evolution of surgery is partial automation, which would ease the cognitive load on the surgeon, making them possible to pay more attention on the critical parts of the intervention. Partial and sequential introduction and increase of autonomous capabilities could provide a safe way towards Surgery 4.0. Unfortunately, autonomy in the given environment, consisting mostly of soft organs, suffers from grave difficulties. In this chapter, the current research directions of subtask automation in surgery are to be presented, introducing the recent advances in motion planning, perception, and human-machine interaction, along with the limitations of the task-level autonomy. 


\section{INTRODUCTION}

Minimally Invasive Surgery (MIS) reshaped surgical practice significantly during the last decades. Contrary to the traditional technique operating through large incisions, MIS is performed through few-centimeter wide ports-so-called keyholes-using laparoscopic instruments, the area of operation is observed on endoscopic camera stream. Smaller incisions offers benefits both for the patient and the hospital, like lower risk of complications, rapid recovery and thus shorter hospital stay. On the other hand, MIS presents new challenges to the surgeons, such as the limited range of motion, different forms of motion, with less Degrees of Freedom (DoF) and also fatigue from weary postures.

Robot-Assisted Minimally Invasive Surgery (RAMIS) was introduced to ease these difficulties. The idea of teleoperated master-slave surgical systems originates from space research: the intervention was to be performed on the patient-in this case an astronaut - by a slave device, controlled by a human surgeon through a master device on Earth (Márton, Szántó, Haidegger, Galambos \& Kövecses, 2017; Takács, Nagy, Rudas \& Haidegger, 2016). The slave side robot arms are equipped by laparoscopic instruments and an endoscopic camera, and copy the movement of the surgeon next to the remote master console, who is able to observe the operation on the endoscopic camera stream.

In the past years, there has been a strong uptake of Computer-Integrated Surgery (CIS) systems, and their current global annual market potential is estimated at $\$ 11.4$ bn, with an expected annual growth of $7 \%$, according to a recent study by LG Electronics. Also, more and more people accept the advancement of surgical robotics, although, the willingness was measured lower in the developed countries

in a recent survey conducted by PricewaterhouseCoopers (PwC) (Fig. 1). A recent Eurobarometer poll found that $26 \%$ of responders would be comfortable with a robot surgeon operating on them ( $2 \%$ up from 2014$)^{1}$, while IEEE found

Figure 1. International acceptance of robotic surgery. A 2017 study by PwC found that there is growing number of people who would embrace these technologies (PricewaterhouseCoopers, 2017) Image credit: $P w C$

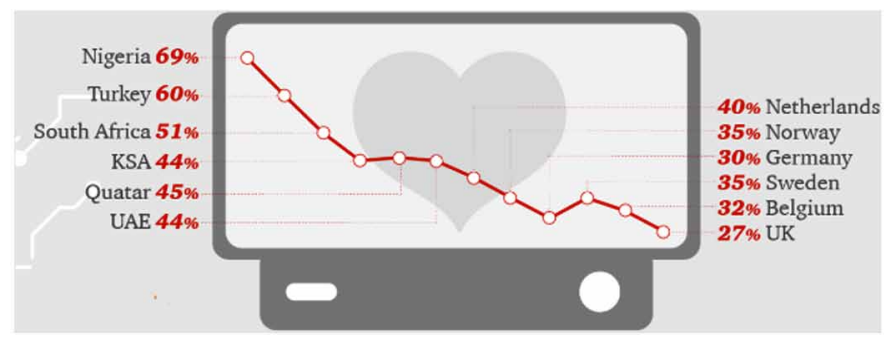


that $62 \%$ of people would be willing to allow an AI driven robot to operate on their children if need be ${ }^{2}$.

However, real remote teleoperation has not become a daily practice, and stalled at the state of research mainly due to the issues caused by time delay, it turned out that teleoperation itself can present a number of benefits. The communication latency — being the biggest issue — can be reduced to a level that is insignificant for the surgeon by placing the master and the slave devices close to each other; in the case of commercial RAMIS, the master and the slave side devices are in the same room. This technique can reduce the fatigue of the surgeon, being able to operate in a more ergonomic, seated position. Furthermore, the movement of the surgeon can be scaled on the slave side - the most delicate movements can be controlled by relatively large hand movements, and hand tremors can also be filtered.

There is no doubt, that the most successful RAMIS device on the market is the da Vinci Surgical System (Intuitive Surgical Inc., Sunnyvale, CA). The $1^{\text {st }}$ generation da Vinci was cleared by the U. S. Food and Drug Administration (FDA) in 2000, and soon became widely used. In 2019 , its $4^{\text {th }}$ generation is available (Fig. 2.), with more than 5300 units installed worldwide, which performed over 1 million procedures last year (Takács et al., 2016).

\section{BACKGROUND}

Many believe that the next necessary step in the evolution of surgery is automation. Already, as of today, the partial automation of surgery is feasible. The workflow of RAMIS procedures frequently contains monotonous and time-consuming elements, automation of such subtasks would ease the cognitive load on the surgeon, making them possible to pay more attention on the critical parts of the intervention (Elek et al., 2017, 2016).

Unfortunately, autonomy in the given environment, consisting mostly of soft tissues, suffers from grave difficulties. Unlike working on rigid-tissues, where exact registration to the instrument is possible, soft tissues are permanently in motion, and highly deformable, thus no pre-computed tool trajectories can be used. A key enabling approach to reduce complexity is dividing the surgical workflow into atomic elements. Identifying surgical subtasks makes it possible to guide the robotic instruments during soft tissue operations, following the decomposed surgical motions. This can be performed on different levels of granularity, and can be used to compile a parameterizable motion library (D. Á. Nagy, Nagy, Elek, Rudas \& Haidegger, 2018; T. D. Nagy \& Haidegger, 2018).

Another challenge of surgical automation is undoubtedly the implementation of perception algorithms usable in the complex human environment. Computer 
Figure 2. The 4 generations of the da Vincy Surgical System; a) da Vinci Classic, b) da Vinci S, c) da Vinci Si and d) da Vinci Xi

Image credit: Intuitive Surgical Inc.

a)

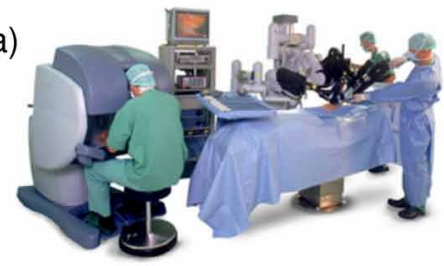

c)

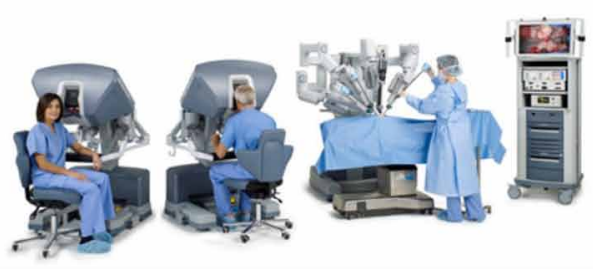

b)

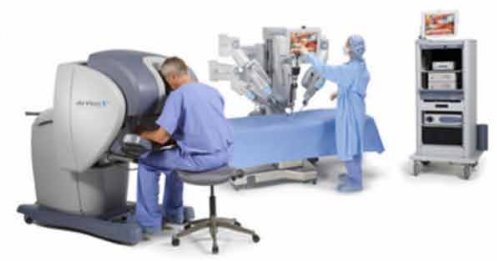

d)

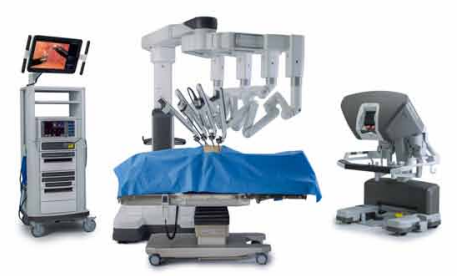

vision suffers from reflections and the fact that the visual features of different organs being very similar, yet it is still the gold standard. New methods relying on palpation emerged in the last years, where force sensors can be utilized e.g., to find the location of tumors or other anatomies/pathologies (Garg et al., 2016; Wei, Arbter \& Hirzinger, 1997; Yamamoto, Vagvolgyi, Balaji, Whitcomb \& Okamura, 2009).

Finally, irrespective of the nature of automation, the supervision of the surgeon is crucial and safety critical during the execution, that requires effective human-machine interaction. The surgeon should be able to parameterize and launch autonomous execution. They also must have the ability observe the area of operation during autonomous execution, and to gain back manual control anytime necessary, or the autonomous algorithm encounters events it cannot solve (Chen, 2016), similarly to the domain of self-driving cars (Takács, Drexler, Galambos, Rudas \& Haidegger, 2018).

In this chapter, the main challenges associated with the development of autonomous surgical robotics are discussed, starting with the objective assessment of capabilities and functions. The above-mentioned aspects of subtask automation in surgery are presented, introducing the recent advances in surgical robot motion planning, perception and human-machine interaction, along with the limitations of the task-level autonomy. 


\section{STANDARDIZATION AND SAFETY CONCERNS}

One of the benefits of international standards is that they may facilitate the safe implementation of new technologies into advanced prototypes and products. When it comes to the field of autonomous surgical robotics, safety is probably the biggest concerns, therefore the major Standards Development Organizations (SDOs) have long started to take action - working towards a safety standard framework for medical and surgical robots (Haidegger, 2019).

\section{Degree of Autonomy of Surgical Equipment}

Roughly 8 years ago the joint International Organization for Standardization and International Electrotechnical Commission (ISO/IEC) Technical Committee (TC) group analyzed the status of surgical robot standardization. Only one major gap was found: the Degree of Autonomy (DoA)_-introduced in ISO 8373:2012 - Robots and robotic devices -- Vocabulary-was not defined properly. Understanding the fact that the proper definition of autonomy and its conjugated forms "autonomous", "automation", or related definitions can be unambiguous, the ISO/IEC joint working group decided to extend the scope of their work to all Medical Electrical Equipment (MEE) or Medical Electrical System (MES) with a DoA (other than zero). The discussion on the topic was concluded in a new Technical Report (TR) IEC/TR 60601-4-1: Medical electrical equipment - Part 4-1: Guidance and interpretation - Medical electrical equipment and medical electrical systems employing a degree of autonomy. The TR recommended to omit such words like "automation" or "automatic" within this robotic standard; DoA was defined instead as "taxonomy based on the properties and capabilities of the MEE or MES related to autonomy".

Derived from the field of industrial automation (Kaber \& Endsley, 2004) and service robotics (Huang, 2008), the TR recommends the parameterization of DoA along four cognition-related functions of a system, which are affecting options of an MES:

- Generate an option: to formulate possible options, based on the result of the monitoring task for achieving predefined goals.

- Execute an option: to carry out the selected option. Robots can typically be active or passive supporters of a surgical task execution.

- Monitor an option: to collect necessary information to perceive the status of MEE or MES, patient, operator or environment. Therefore, signals beyond the internal (proprioceptive) control signals of the robot.

- Select an option: to decide on a particular option from the pool of generated. 
$D o A=\mathcal{F}\{G|E| M \mid S\}$,

where the overall DoA metric is normed sum of the four function of the system assessed on a linear scale, 0 meaning fully manual and 1 fully autonomous.

The objective assessment of the DoA of a system can be utilized along the four described functions, each can be performed by a human or by a computer. The DoA of the system is defined on a scale from 0 to 1 ; DoA $=0$ means "no autonomy", and the highest DoA represents "full autonomy". The low-level electronic and computational functions of MEE or MES, like communication or motor control, are excluded from this definition, as the term "no autonomy" is meant on the system level. Also, a classification of DoA can be given at different levels of granularity, depending on the level where are those functions implemented.

Autonomous execution is already present is surgery (especially in image-guided surgery), however decision making (selecting) is mostly done by human experts (Haidegger, 2019). On the other hand, computer systems are more capable in monitoring task compared to humans, hence most critical processes happen at a faster scale humans can perceive; this is the safety concept of Situation Awareness (SA).

The TR offers various alternatives for DoA assessment; the most applicable one, based on industrial automation, defines 10 levels of DoA (Table 1). Practically, during the risk management of a surgical robot (most commonly according to ISO 14971 - Application of risk management to medical devices), DoA should also be taken into account; DoA does not necessarily correlates with the level of risk, but it may impact risk management gravely. In case of an error or malfunction, the takeover of the human operator may be necessary. Due to loss of SA the operator may not be able to control the situation properly, thus the DoA determines the handling of the hazards. At lower DoA, the responsibility can be shared between the human and the robot; at higher DoA malfunctions presents critical hazards.

\section{Basic Safety and Essential Performance of Surgical Robots}

From the aspect of the user (and also of the manufacturer), avoiding and managing any kind of failure (software, hardware, communication, system-level) is critical. In the past three decades, 33 documented casualties were caused by industrial robots, which is still only giving $0.0005 \%$ of all work-related deaths (Delvaux, 2016) None of the surgical robotic cases are included in this statistics. In surgical roboticsespecially in RAMIS - the human surgeon takes full liability for the outcome of the intervention (as ruled in all juridical cases up to now).

With the increase of DoA, risk mitigation and management becomes crucial. Methodologies to support the safety of design and development of robotic devices 
Table 1. Descriptive classification of degree of autonomy adapted from (Kaber \& Endsley, 2004) based on IEC/TR 60601-4-1. H: the human operator performs the given function. $C$ : the computer-driven system performs the given function

\begin{tabular}{|c|c|c|c|c|c|}
\hline DoA & Description & Monitor & Generate & Select & Execute \\
\hline 1.0 & $\begin{array}{l}\text { Full Manual (FM): No autonomy involved. The operator } \\
\text { performs all tasks including monitoring the state of the system, } \\
\text { generating performance options, selecting the option to perform } \\
\text { (decision making) and executing the decision made, i.e, } \\
\text { physically implementing it. }\end{array}$ & $\mathrm{H}$ & $\mathrm{H}$ & $\mathrm{H}$ & $\mathrm{H}$ \\
\hline 2. 1 & $\begin{array}{l}\text { Teleoperation (TO): The equipment assists the operator with } \\
\text { the execution of the selected action, although continuous } \\
\text { operator control is required. The operator performs all tasks, } \\
\text { including monitoring the state of the equipment, generating } \\
\text { options, selecting the desired option and execution of it. } \\
\text { (Master-Slave teleoperation.) Note: traditional robotics } \\
\text { standards consider teleoperation as zero DoA. }\end{array}$ & $\mathrm{H} / \mathrm{C}$ & $\mathrm{H}$ & $\mathrm{H}$ & $\mathrm{H} / \mathrm{C}$ \\
\hline 3.2 & $\begin{array}{l}\text { Pre-Programmed Execution (PE): The operator generates } \\
\text { and selects the options to be performed without any analysis or } \\
\text { selection by the equipment. Note: traditional robotic standards } \\
\text { considered this as "autonomous" or "automatic" operation. }\end{array}$ & $\mathrm{H} / \mathrm{C}$ & $\mathrm{H}$ & $\mathrm{H}$ & $\mathrm{C}$ \\
\hline 4.3 & $\begin{array}{l}\text { Shared Decision (SD): Both the operator and the equipment } \\
\text { generate possible decision options. The operator retains full } \\
\text { control over the selection of which option to execute. Both the } \\
\text { operator and the equipment participate in the execution. }\end{array}$ & $\mathrm{H} / \mathrm{C}$ & $\mathrm{H} / \mathrm{C}$ & $\mathrm{H}$ & $\mathrm{H} / \mathrm{C}$ \\
\hline 5.4 & $\begin{array}{l}\text { Decision Support (DS): Me equipment generates a list of } \\
\text { decision options, which the operator can select from, or the } \\
\text { operator may generate alternative options. Once the human } \\
\text { has selected an option, it is turned over to the equipment to } \\
\text { execute it. }\end{array}$ & $\mathrm{H} / \mathrm{C}$ & $\mathrm{H} / \mathrm{C}$ & $\mathrm{H}$ & $\mathrm{C}$ \\
\hline 6.5 & $\begin{array}{l}\text { Blended Decision (BD): The equipment generates a list of } \\
\text { decision options, which it selects from and executes if the } \\
\text { operator consents. The operator may also generate and select an } \\
\text { alternative option; the equipment will then execute the selected } \\
\text { action. BD represents a high-level decision support system that } \\
\text { is capable of selecting among alternatives as well as executing } \\
\text { the selected option. }\end{array}$ & $\mathrm{H} / \mathrm{C}$ & $\mathrm{H} / \mathrm{C}$ & $\mathrm{H} / \mathrm{C}$ & $\mathrm{C}$ \\
\hline 7.6 & $\begin{array}{l}\text { Guided Decision (GD): The equipment presents a set of } \\
\text { actions to the operator. The operator's role is to select from this } \\
\text { set, he/she cannot generate any other additional options. The } \\
\text { equipment will fully executes the selected action. }\end{array}$ & $\mathrm{H} / \mathrm{C}$ & $\mathrm{C}$ & $\mathrm{H}$ & $\mathrm{C}$ \\
\hline 8.7 & $\begin{array}{l}\text { Autonomous Decision (AD): The equipment selects the best } \\
\text { option and executes it, based upon a list of alternatives it } \\
\text { generates (this list can be augmented by alternatives suggested } \\
\text { by the operator). }\end{array}$ & $\mathrm{H} / \mathrm{C}$ & $\mathrm{H} / \mathrm{C}$ & $\mathrm{C}$ & $\mathrm{C}$ \\
\hline 9.8 & $\begin{array}{l}\text { Operator Monitoring }(\mathbf{O M}) \text { : The equipment generates } \\
\text { options, selects the option to implement and executes it. The } \\
\text { operator monitors the equipment and intervenes if necessary. } \\
\text { Intervention places the human in the role of making a different } \\
\text { option selection. During the procedure there may be decision } \\
\text { making points that will be decided by the equipment. }\end{array}$ & $\mathrm{H} / \mathrm{C}$ & $\mathrm{C}$ & $\mathrm{C}$ & $\mathrm{C}$ \\
\hline 10.9 & $\begin{array}{l}\text { Full Autonomy (FA): The equipment carries out all actions. } \\
\text { The operator does not intervene except to e-stop the equipment } \\
\text { (which is a general requirement). }\end{array}$ & $\mathrm{C}$ & $\mathrm{C}$ & $\mathrm{C}$ & $\mathrm{C}$ \\
\hline
\end{tabular}

were published by various groups, like the generic Hazard Identification and Safety Insurance Control (HISIC) policy that has been applied to several robotic systems (Fei, Ng, Chauhan \& Kwoh, 2001). 
In 2015 the ISO/IEC TC 62/SC 62D joint committee started a discussion on the minimum requirements for a practical degree of safety for surgical robots; the results are planned to be published in 2019 as IEC 80601-2-77: Particular requirements for the basic safety and essential performance of robotically assisted surgical equipment. The standard collects all relevant thermal and mechanical hazards, along with the required usability trials. It also defines the basic types of surgical robots and their relevant components. In terms of RAMIS, the focal points of the standard are:

- Robotic surgical instruments.

- The patient-side part of the robot.

- The operator-side part of the robot.

- The endoscope holder (if any).

The same committee also presented a standard, focusing on the hazards related to loss of SA, namely IEC/CD 80601-2-78: Particular requirements for the basic safety and essential performance of medical robots for rehabilitation, compensation or alleviation of disease, injury or disability. This standard defines SA as "the operator's perception, comprehension and prediction of a robot's behavior in its environment". SA is a key factor in tasks where human supervision or interaction with the robot is necessary to reduce risk. The standard states that the manufacturers will have to include necessary SA information for their future medical robotic systems. The quality of the Human-Robot Interface (HRI) is essential to keep SA; if the critical information is forwarded to the human operator through an adequate way, e.g., sound alerts, that may highly increase SA. Furthermore, using AI methods, the system may also offer decision support to handle complex situations (Chen, 2016).

It is believed by the community, that upcoming standards would focus more on the safety of the patients and the improvement of the treatments rather than technical metrics, and willing to continue work aiming that goal.

\section{Level of Autonomy for Surgical Robots}

Although the standards, mentioned above are fundamental for the assessment of the capabilities of surgical robotic systems, they are not definite enough to present taxonomy to generally assess the development phases of surgical robotics, or to perform benchmarking. Surgical robotic systems need to be categorized based on their advancement, relative in the field. A gradual mapping was presented in (Yang et al., 2017), to classify the autonomous capabilities of surgical robots. Some earlier work suggested to put the HRI into the center of the classification, defining a 0-7 scale (Rosen, 2013). Similar concepts are also presented in the field of self-driving; in (Takacs, Rudas, Bosl \& Haidegger, 2018) a 6-grade scale was introduced for 
autonomous vehicles. At higher levels of autonomy, the role of the robot is still argued; the robot is not only a medical device anymore, but it also practices medicine, which is entirely different from the viewpoint of regulatory. FDA, for example, regulates medical devices, but not the practice of medicine.

The mapping of (Yang et al., 2017) has one fundamental problem in the middle ranges Level of Autonomy (LoA), where the most of the current autonomous capabilities would fall into: this mapping offers no metric to determine the level of human supervision required. The role of SA may be crucial to distinguish the cognitive level up to which the human may be able and shall be allowed to perform take-over; described as human-on-the-loop control (Mohamed, Hossny, Nahavandi, Dalvand \& Asadi, 2018).

It is evident human supervision dependent scale in any mapping of autonomy. The sensory and processing bandwidth capabilities of humans are limited, thus the loss of SA would mean that the conditional enabling of a robot functionality, or a supervising its actions require SA, and only the cognitive time horizon is different, in which the human can react.

Coherent to the current standardization efforts, yet fitting to the commonly used terms, the following scale of LoA is suggested (Haidegger, 2019) (Fig. 3.):

- LoA 0 - No Autonomy: All system-level functions (generating, selecting, executing and monitoring actions) are performed by the human operator. Technically it means that during the surgery no active robotic equipment is used, thus it may be considered identical to a non-robotic case.

- LoA 1 - Robot Assistance: The surgical robot performs specific, low level functions only. E.g., teleoperated systems, tremor filtering, minor safety features.

- LoA 2 - Task-level Autonomy: The system is trusted to complete certain tasks or sub-tasks in an autonomous manner. E.g., image-guided bone drilling, wound closure. It may only happen for a short instance.

- LoA 3 - Supervised Autonomy: The system can autonomously complete large section of a surgical procedure, while making low-level cognitive decisions. All actions are performed under human supervision, assuming the operator's situation awareness.

- LoA 4 - High-Level Autonomy: The robotic system executes complete procedures based on human-approved surgical plans, while the human only has the capability to emergency stop (e-stop) the procedure. The robot shall be able to complete the task even if the human fails to respond appropriately to a request to intervene.

- LoA 5 - Full Autonomy: A full-time performance of the robotic system, handling all environmental and adverse conditions. The system succeeds in 
scenarios where even the best human operator would fail, therefore there will be no need for a human fallback option.

Unlike DoA, this LoA definition is empirical, focusing on the key enabling robot capabilities of a system. Full autonomy of surgical robots is still belongs to science fiction, however, several techniques based on AI are being under intensive research by various research groups (Richter, Orosco \& Yip, 2019). Many believe, that, similarly to domain of self-driving vehicles, the market will suddenly get interested in autonomy, as LoA 3 (Supervised Autonomy) becomes reliable and gain currency.

\section{THE GRANULARITY LEVELS OF SURGERY}

To gain a better understanding of surgery, including the workflow or the movement of the surgeon, hierarchic decomposition may be an expediential instrument (Gao et al., 2014; MacKenzie, Ibbotson, Cao \& Lomax, 2001; Nagyné Elek \& Haidegger, 2019; Vedula et al., 2016). This deeper knowledge can fuel automation, and also the development of surgical ontologies - the machine-readable representation of surgical knowledge (D. Á. Nagy, Nagy, et al., 2018).

The literature of the field presents various definitions on some levels of granularity, however, no consistent definitions are yet compiled for the whole domain. For the decomposition of the surgeon's motion, and also for the purpose of partial automation, the precise formulation of such definitions is essential. Thus, according to the current state of the art, the granularity levels of surgery is defined by the authors as follows (Fig. 4.):

1. Operation: The entire invasive part of the surgical procedure.

2. Task: Well delimited surgical activity with a given high-level target/goal to achieve.

3. Subtask: Circumscribed activity segments that accomplish specific minor landmarks in completing the surgical task.

4. Surgeme: An atomic unit of intentional surgical activity resulting in a perceivable and meaningful outcome.

5. Motion primitive: General elements of motion patterns, that can be directly translated into robot commands.

In the current studies, which target surgical automation, subtask level activities are chosen most frequently. The completion of subtasks usually results in the accomplishment of a specific milestone, that fits the scope of partial automation quite well. The next level downwards - the level of surgemes-contains elements, 
Figure 3. The proposed 6-stage classification for assessing the autonomous capabilities of surgical robots. The concept of Level of Autonomy follows the ISO/ IEC standardization framework, determining LoA based on the human versus robotic functions of the system (Haidegger, 2019)

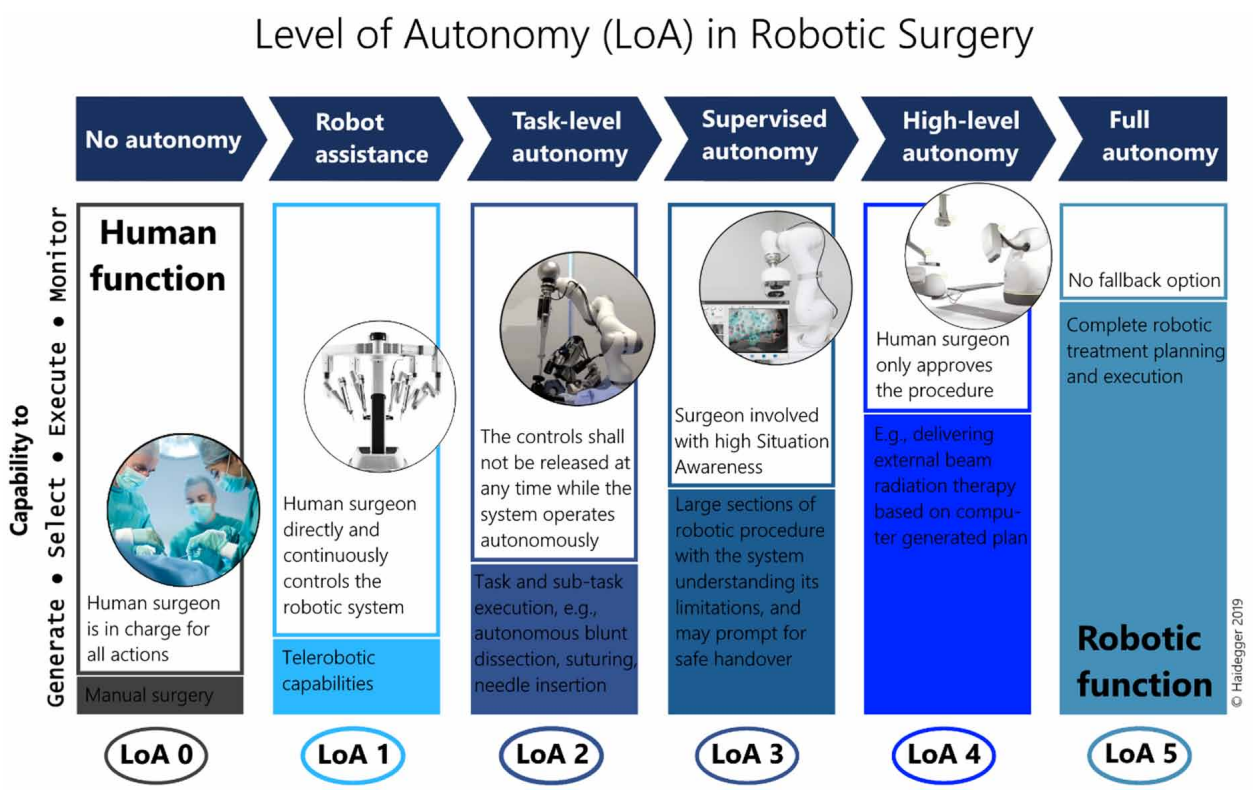

which are universal to various subtask, making possible the implementation of an universal motion library of parameterizable surgemes for automation.

Additionally, for the separation of subsequent surgemes from one another, certain events and features are defined. One, and probably the most obvious one is the shape of motion, which distinguishes e.g., grasping and releasing of an object. Tissue interaction during the surgeme execution is also crucial; the tool can be moving freely in the patient's body, or can grasp a free or and anchored piece of tissue. The change in environmental interaction usually means transitioning between different surgemes. The final feature listed here is the instrumentation-e.g., cutting can be executed using scissors, but scissors must not be used for grasping-, as the change of instruments always occurs at surgeme transitions (McKinley et al., 2016).

\section{RESEARCH PLATFORMS}

To realize the data collection and processing for the above, complete research platforms had to be built and constructed, bringing computer technology to the 
Figure 4. Overview of surgical motion's granularity levels. Mapping of an example, Laparoscopic Cholecystectomy procedure onto different granularity levels (Elek et al., 2017; T. D. Nagy \& Haidegger, 2018)

\begin{tabular}{|c|c|c|c|c|}
\hline $\begin{array}{l}\text { Level of } \\
\text { granularity }\end{array}$ & Definition & $\begin{array}{l}\text { Time } \\
\text { span }\end{array}$ & $\begin{array}{c}\text { Com- } \\
\text { plexity }\end{array}$ & Example \\
\hline Operation & $\begin{array}{l}\text { The entire invasive part of } \\
\text { the procedure. }\end{array}$ & 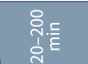 & 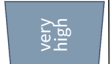 & $\begin{array}{c}\begin{array}{c}\text { Laparoscopic } \\
\text { cholecystectomy }\end{array} \\
\end{array}$ \\
\hline Task & 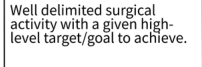 & 织最 & $\frac{\text { 兽 }}{\sqrt{2}}$ & 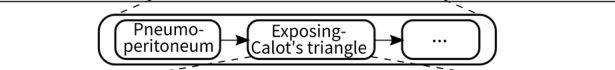 \\
\hline Subtask & 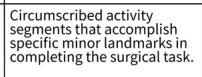 & 궁를 & 离 & 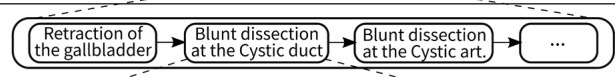 \\
\hline Surgeme & 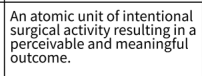 & 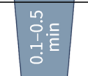 & $\frac{z}{\underline{z}}$ & $\begin{array}{c}\text { Perform } \\
\text { dissecting motion }\end{array}$ \\
\hline $\begin{array}{l}\text { Motion } \\
\text { primitive }\end{array}$ & 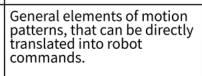 & 늠 & 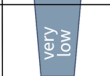 & $\begin{array}{c}\left(\begin{array}{c}\text { Penetrate } \\
\text { connective tissue }\end{array}\right.\end{array} \rightarrow \begin{array}{c}\begin{array}{l}\text { Open the } \\
\text { dissector }\end{array} \rightarrow \text { Remove } \\
\text { the dissector }\end{array}$ \\
\hline
\end{tabular}

operating room. On the academic research side, the Robot Operating System (ROS) platform is widely used in the research of robotics, and also preferred by many in the medical robotics domain; most of the research centers, working on the two dominant research platforms presented below, rely on ROS. ROS is undoubtedly a powerful, modular tool with already implemented solutions for most of the frequently occurring problems of the field, such as stereo-camera calibration or acquisition of sensory data. Its more robust version, ROS-Industrial is also available (https:// rosindustrial.org).

\section{Da Vinci Research Kit}

Approximately 5 years ago, when the $1^{\text {st }}$ generation da Vinci robots (da Vinci classic) was sent to retirement due to the discontinued service and supply, the old systems found another purpose. Those systems were still functional and could be utilized in applications more tolerant to malfunctions. At the Johns Hopkins University the development of a research platform for those robots- the Da Vinci Research Kit (DVRK) — was concluded, and only in a few years, an active community was built with more than 35 setups worldwide (Kazanzides et al., 2014).

DVRK is a fully open-source platform, consists of custom hardware and software elements, in order to open the possibility of programming the da Vinci arms. The controllers - developed to operate the arms — built on custom boards: an IEEE-1394 FPGA board for computational power and low latency communication, and a Quad Linear Amplifier (QLA) for high-frequency low-level robot control. The controllers are connected to PC using IEEE 1394a (FireWire). On the PC side, the opensource cisst libraries (Kazanzides, 2005) are reliable for the handling of FireWire 
communication and the mid-level control of the robot. The cisst libraries offer the functionality to program the arms themselves. Additionally, cisst is also interfaced with ROS, which interface is currently used to program the da Vinci arms at more than half of the DVRK locations (Z. Chen, Deguet, Taylor \& Kazanzides, 2017).

\section{The RAVEN Platform}

The RAVEN-I platform was originally developed at the University of Washington in the mid-2000s, aiming for space use, and other specific application areas. Hence most surgical robots were bulky, and used dominantly in the operating room. RAVEN-I was to be a new, lighter, portable and still durable surgical robot, with possibility to be used on the field. After it has proved its versatility and durability in a number of experiments, e.g., in a trial on an underwater research station, as a part of the NASA's NEEMO program, its research potential was also soon discovered. In the beginning of 2010s, the University of Washington Biorobotics Lab and the University of California Santa Cruz Bionics Lab developed an updated design of the system, named RAVEN-II. Later, Applied Dexterity http://applieddexterity. com/about/history) was formed to support the RAVEN community, and also the development of the RAVEN-III platform was started. In 2019, there are 16 RAVEN sites worldwide within the cutting-edge research of surgical robotics.

The research platform is fully open-source, consists of two $3 \mathrm{DoF}$ positioning arms, with 4 DoF attachable instruments - similar to the da Vinci. Like the DVRK, the system is Linux-based, and uses ROS interface for programming (Hannaford et al., 2013).

\section{RECENT TRENDS IN AUTOMATING SURGERY}

Currently, partial (or conditional) automation is the most intensively research domain of surgical automation. The workflow of RAMIS interventions often contain subtask elements, that are time-consuming and monotonous for the surgeon to complete, such as knot-tying or blunt dissection. Partial automation - the automation of such subtasks - may reduce the cognitive load and fatigue on the human surgeon, making possible them to pay more attention on the critical parts of the operation (D. Á. Nagy, Rudas \& Haidegger, 2018).

The technological advancements of the last few years in the domain of deep learning or mechatronics offer a rising potential on the research of surgical subtask automation (Karoly, Kuti \& Galambos, 2018). Several of those are under intensive development or implemented already by research groups worldwide (Table 2.). 
Table 2. List of surgical subtasks from the aspect of automation. FRS: Fundamentals of Robotic Surgery (Florida Hospital Nicholson Center, Celebration, FL, STAR: Smart Tissue Autonomous Robot)

\begin{tabular}{|c|c|c|c|c|c|c|}
\hline Subtask & Platform & $\begin{array}{c}\text { Sensor } \\
\text { Integration }\end{array}$ & $\begin{array}{l}\text { Experimental } \\
\text { Environment }\end{array}$ & Complexity & $\begin{array}{c}\text { Clinical } \\
\text { Relevance }\end{array}$ & Reference \\
\hline shape cutting & DVRK & $\begin{array}{l}\text { stereo } \\
\text { camera }\end{array}$ & $\begin{array}{l}\text { gauze patch, } \\
\text { FRS Dome }\end{array}$ & medium & high & $\begin{array}{l}\text { (Murali et } \\
\text { al., 2015) }\end{array}$ \\
\hline suturing & DVRK & $\begin{array}{l}\text { stereo } \\
\text { camera }\end{array}$ & $\begin{array}{l}\text { silicone, foam, } \\
\text { FRS Dome }\end{array}$ & high & high & $\begin{array}{l}\text { (Sen et al., } \\
2016)\end{array}$ \\
\hline palpation & DVRK & force sensor & $\begin{array}{l}\text { special silicone } \\
\text { phantom, FRS } \\
\text { Dome }\end{array}$ & medium & medium & $\begin{array}{l}\text { (Garg et } \\
\text { al., 2016; } \\
\text { Nichols \& } \\
\text { Okamura, } \\
\text { 2013) }\end{array}$ \\
\hline $\begin{array}{l}\text { tumor } \\
\text { palpation and } \\
\text { resection }\end{array}$ & $\begin{array}{l}\text { DVRK, } \\
\text { RAVEN }\end{array}$ & force sensor & $\begin{array}{l}\text { special silicone } \\
\text { phantom, FRS } \\
\text { Dome }\end{array}$ & high & medium & $\begin{array}{l}\text { (McKinley } \\
\text { et al., 2016) }\end{array}$ \\
\hline debridement & DVRK & $\begin{array}{l}\text { stereo } \\
\text { camera }\end{array}$ & tiny objects & medium & high & $\begin{array}{l}\text { (Murali et } \\
\text { al., 2015; } \\
\text { Seita et al., } \\
\text { 2017) }\end{array}$ \\
\hline $\begin{array}{l}\text { bowel } \\
\text { anastomosis }\end{array}$ & STAR & 3D camera & porcine bowel & high & high & $\begin{array}{l}\text { (Shademan } \\
\text { et al., 2016) }\end{array}$ \\
\hline $\begin{array}{l}\text { blunt } \\
\text { dissection }\end{array}$ & DVRK & $\begin{array}{l}\text { stereo } \\
\text { camera }\end{array}$ & $\begin{array}{l}\text { sandwich- } \\
\text { like silicone } \\
\text { phantom }\end{array}$ & medium & high & $\begin{array}{l}\text { (Elek et al., } \\
\text { 2017; D. Á. } \\
\text { Nagy, Nagy, } \\
\text { et al., 2018) }\end{array}$ \\
\hline $\begin{array}{l}\text { tissue } \\
\text { retraction }\end{array}$ & DVRK & $\begin{array}{l}\text { stereo } \\
\text { camera }\end{array}$ & $\begin{array}{l}\text { silicone } \\
\text { phantom }\end{array}$ & low & high & $\begin{array}{l}\text { (T. D. Nagy, } \\
\text { Takács, } \\
\text { Rudas \& } \\
\text { Haidegger, } \\
\text { 2018) }\end{array}$ \\
\hline peg transfer & DVRK & $\begin{array}{l}\text { stereo } \\
\text { camera }\end{array}$ & $\begin{array}{l}\text { training } \\
\text { phantom }\end{array}$ & medium & low & $\begin{array}{l}\text { (T. D. } \\
\text { Nagy \& } \\
\text { Haidegger, } \\
\text { 2018) }\end{array}$ \\
\hline
\end{tabular}

One of the first successful projects in the domain - the work of UC Berkeley AUTOLAB and Center for Automation and Learning for Medical Robotics (CALMR) - was presented (Fig. 5.) (Murali et al., 2015), with not one, but two surgical subtasks completed autonomously in phantom environment, using the DVRK: multilateral (using at least two arms) debridement (Fig. 6. a-b) and shape cutting (Fig. 6. c). In this work, the learning by observation approach was used: human motion patterns were recorded and segmented, and then those patterns were used to generate robot trajectories during autonomous execution. The motion segments 
were, e.g., in case of debridement: motion, penetration, grasping, retraction and cutting. In order to autonomously execute the motion segments, a state-machine was compiled for each subtask. The state machine required parameters for the motion segments for execution, e.g., the height of lifting motion in case of retraction. The parameters were determined empirically, using binary search methodology. After each motion segment was parameterized and tested individually, the whole state machine was tested again, and the parameters updated, if necessary (Fig. 5.). The positions of the targets were estimated by computer vision, two pairs of stereo cameras were used to observe the field of operation. The debridement targets and the circle for cutting were detected in the images, and robot's trajectories were translated based on the $3 \mathrm{D}$ coordinates of the detected objects. To evaluate the autonomous execution, autonomous debridement was executed 10 times with 5 targets each, and shape-cutting were performed 20 times. The repeatability of the subtasks (ratio of successful trials) was respectively $96 \%$ and $70 \%$.

Another work of the same research group (McKinley et al., 2016), aimed at autonomous multilateral tumor resection based on palpation in phantom environment (Fig. 6.d). To achieve the completion of this series of subtask, custom-built instruments were designed, attachable to end effector of the da Vinci: a palpation probe, a scalpel and a fluid injector. Also, a state machine was compiled to execute the series of subtasks: scan the phantom by palpation and localize the hard inclusion, making of the incision, removal of the inclusion (debridement) and fluid injection to close the wound. To evaluate the performance of the system, 10 end-to-end trials were performed, with overall $50 \%$ success rate. In 2 of the trials, the tumor location was estimated incorrectly, another 2 times retraction failed, and in 1 trial the tumor was not fully resected, which shows the challenges given the complexity of any surgical procedure. The authors asserted that the performance could be enhanced by visual feedback and are planned to include computer vision features in the future.

Suturing is probably the most intensively researched subtask of RAMIS, it occurs quite frequently in the workflow of surgical interventions, yet extremely time-consuming for the surgeon, and challenging for automation. Suturing has two, highly difficult aspects: needle guidance through the tissue on a given trajectory, and also thread manipulation, especially during knot-tying (Gao et al., 2014; Vedula et al., 2016). In (Sen et al., 2016), another work of the UC Berkeley Automation Lab, a solution for the former one is presented. To precisely grasp the needle, a positioning adapter (Suture Needle Angular Positioner-SNAP) was designed, that itself achieved a 3-folds error reduction in needle pose. The needle position was estimated using camera image, the needle size, trajectory, and control parameters using were optimized using sequential convex programming. During the trials, the system was able to complete $86 \%$ of attempted suture throws successfully. 
Figure 5. The learning by observation process used to compile state machines for autonomous subtask execution.

Image credit: (Murali et al., 2015)

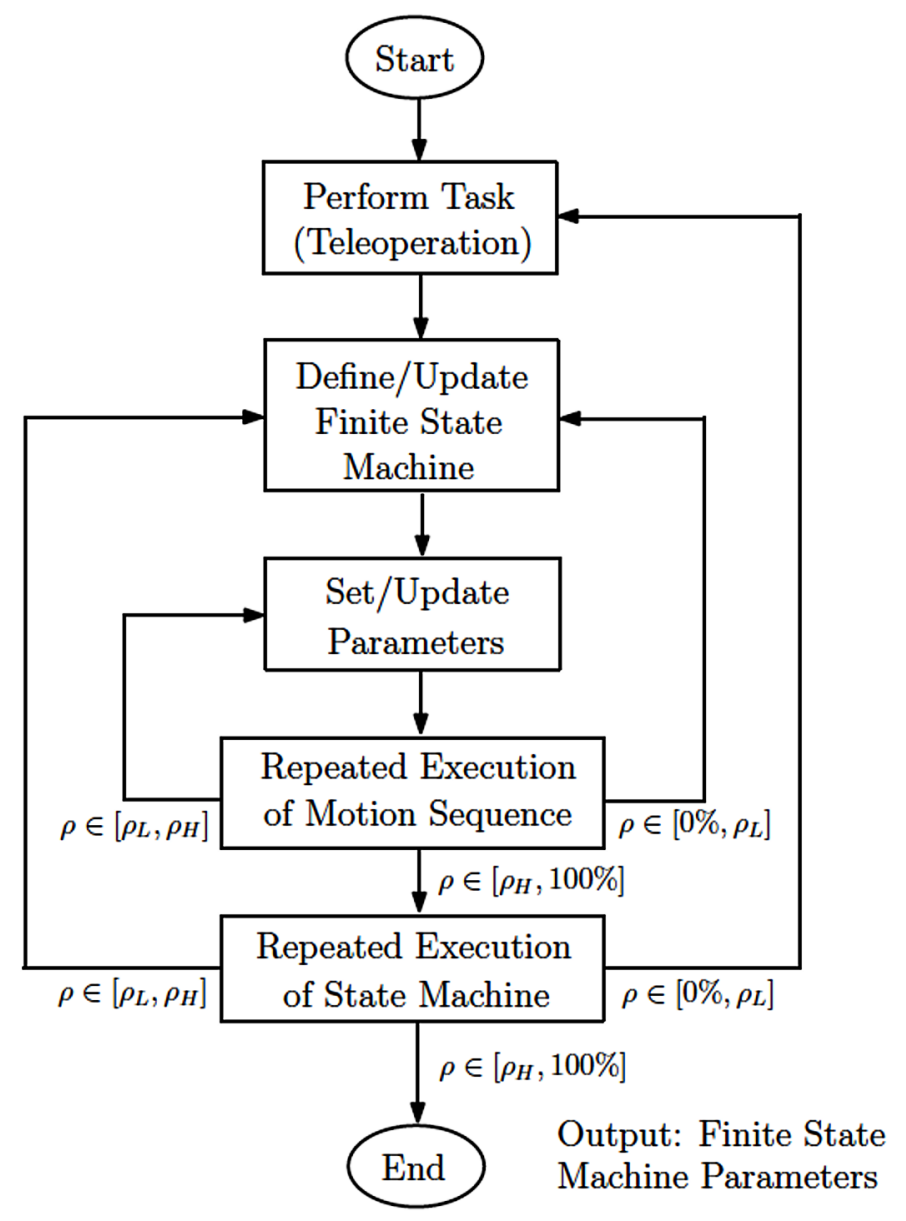

Implementation of autonomous subtasks on cable-driven robots, like the da Vinci or the RAVEN can be challenging due to the their inherent non-linearities. The inaccurate robot positioning causes no issue in teleoperation, as the human surgeon, who follows the tool position on the endoscopic camera stream, is part of the control loop. However, in the case of the automation of position-critical subtasks those inaccuracies can easily cause failures. In (Seita et al., 2017) a two-phase calibration method was presented, to decrease position errors of cable-driven surgical robots, using deep neural network and random forest techniques. By precise calibration, the debridement subtask was automated with $94.5 \%$ success rate (6. e). 
Figure 6. Recently automated surgical subtasks. a-c) Multilateral cutting, d) tumor palpation and e) resection, debridement.

Image credit: (McKinley et al., 2016; Murali et al., 2015; Seita et al., 2017)
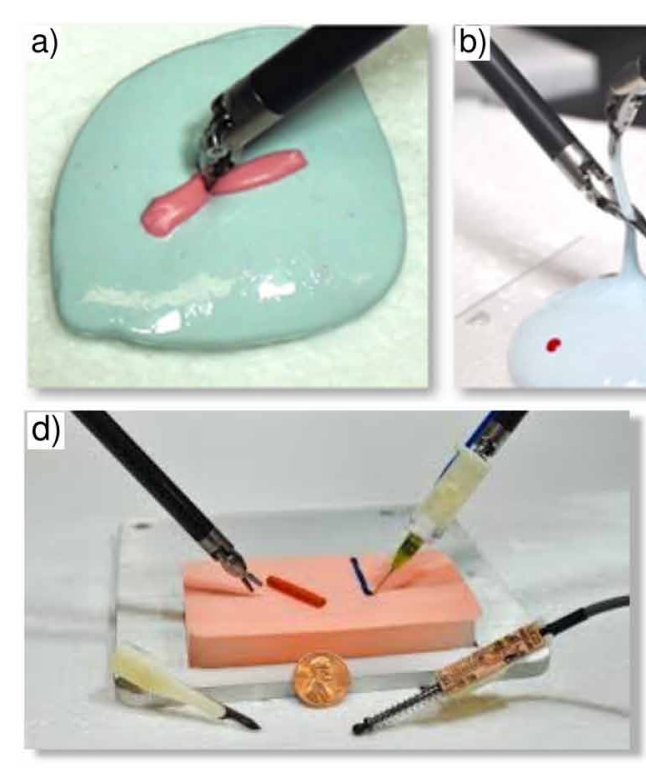
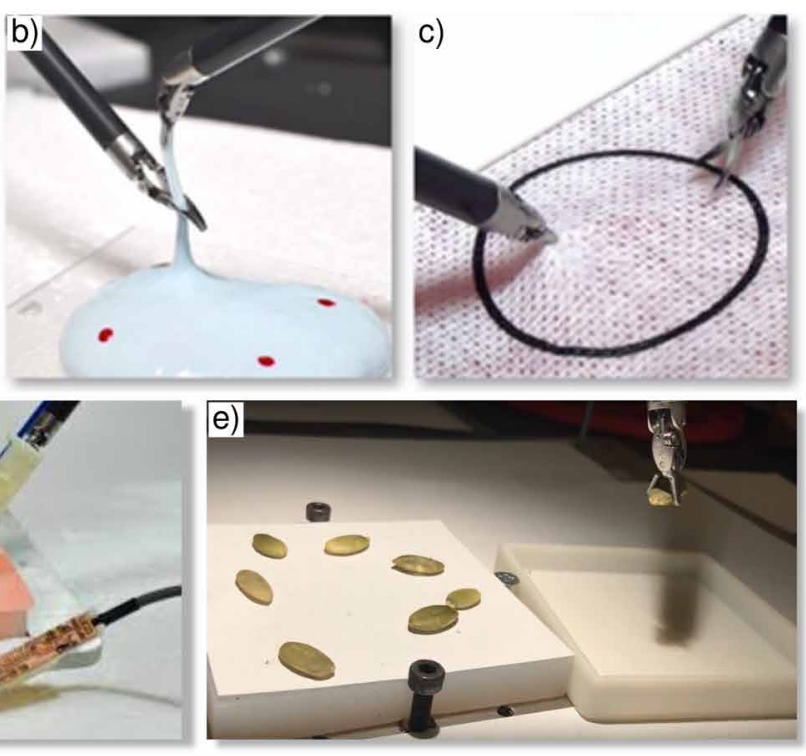

Blunt dissection automated in phantom environment was presented in (Elek et al., 2017). Blunt dissection is used to separate loosely connected layers of tissue, without harming sensitive components in between with sharp object (Fig. 7.). This autonomous approach is also relies on stereo camera, and built by the software framework developed by the authors (T. D. Nagy \& Haidegger, 2018). This modular, ROS-based framework contains a motion library; a set of parameterizable surgemes, universal to a various subtasks. Additionally to blunt dissection, this framework was also used to implement soft tissue retraction (T. D. Nagy, Takács, et al., 2018) and a surgical training exercise, peg transfer (T. D. Nagy \& Haidegger, 2018).

\section{SOLUTIONS AND RECOMMENDATIONS}

With the growing number of robotic applications, new challenges also arise, for which autonomous robot capabilities may be able to provide a solution (Kaplan, Nichols \& Okamura, 2016). Nevertheless, the widening adoption of service robots in the surgical domain also increases the incidence of malfunctions, potential injuries and damage. As a consequence, litigation fears are escalating for companies developing new types of robots Parallel, urgency is growing to have international 
Figure 7. Motion primitives of the blunt dissection subtask. a) The surgical instrument (large needle driver) moves to the dissection target; $b$ ) the robot pushes the instrument into the phantom; $c$ ) the instrument is opened; d) the robot pulls out the instrument. Image credit: (Elek et al., 2017)
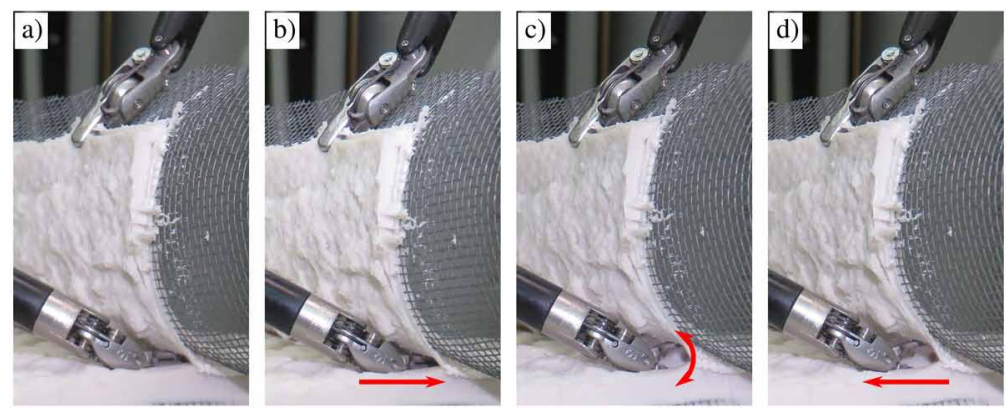

safety standards published to allow new robots to get certified in a transparent way to operate in complex, real-world scenarios.

The market has well understood the need for rigorous development methods and stringent testing. Representing this, the funding of the surgical robotics projects has risen significantly (to the extremes of Auris Health, which collected $\$ 733$ million venture capital money for development and early sales). There is a stronger need nowadays towards incremental advancement in the field, which is better suited for international standardization as well.

\section{FUTURE RESEARCH DIRECTIONS}

CIS and RAMIS within still requires a lot of fundamental research to deliver reliable solutions to the most urging open medical needs. It is remarkable that over $1 \mathrm{~m}$ RAMIS interventions were performed in 2018, yet this is still a fraction of the global annual $300 \mathrm{~m}$ surgeries. The rise of robotic MD training is obviously leading to the wider spread of robotic procedures, and will eventually resolve currently existing resistance among surgeons (Sándor et al., 2010). Given the popularity of open source initiatives (such as ROS-Industrial), software development pathways have become streamlined, successful FDA clearances have been backed by toolkits such as 3D Slicer (https://www.slicer.org/) and PLUS (Lasso et al., 2014).

As it is already happening, smaller scale robots, such as capsules, natural orifice and nano-size robots represent a rising future domain (Forbrigger et al., 2019; Li, Esteban-Fernández de Ávila, Gao, Zhang \& Wang, 2017; Ma, Song, Chiu \& Li, 2019), however, these will fundamentally change the clinical workflow, therefore 
cannot be handled together with the current surgical robots regarding standardization. Already the locomotion capabilities of an active capsule robot require significant autonomous functions from the system, as such are now being tested by many companies, and human clinical trials are underway.

In the meanwhile, capturing the essence of human surgery remains a significant challenge for computer systems. Ontologies and surgical process models are believed to serve efficiently the current automation needs for certain more simple sub-tasks at LoA 3 and presumably LoA 4 (Olszewska et al., 2018). Most of the publicly disclosed projects are in the stage of Technology Readiness Level (TRL) 4-6, in the research and development phase.

\section{CONCLUSION}

We are witnessing the age of robotics and AI, and even the most challenging domains, such as human surgery, are receiving substantial support and uptake by autonomous capabilities. It is evident that safety has the key role in the field of medical robotics, and therefore current research and development efforts mostly address just elements of the complex challenge. In this chapter, the autonomy of surgical robots was reviewed, providing an insight into the current standardization landscape and offering some practical tools for system capability assessment. Lower and middle level of autonomy system prototypes and development projects were reviewed which aim at autonomous subtask level execution. Current major efforts, trends and possible future directions were presented

\section{ACKNOWLEDGMENT}

This work was partially supported by ACMIT (Austrian Center for Medical Innovation and Technology), which is funded within the scope of the COMET (Competence Centers for Excellent Technologies) program of the Austrian Government. Partial support of this work comes from the Hungarian State and the European Union under the EFOP-3.6.1-16-2016-00010 project. T. D. Nagy and T. Haidegger are supported through the New National Excellence Program of the Ministry of Human Capacities. T. Haidegger is a Bolyai Fellow of the Hungarian Academy of Sciences. The grammatical finalization of the chapter was supported by the V4+ACARDC - CLOUD AND VIRTUAL SPACES grant. 


\section{REFERENCES}

Chen, S.-Y. (2016). Study on situation awareness for medical robots. National Taipei University of Technology. [Technical Report]

Chen, Z., Deguet, A., Taylor, R. H., \& Kazanzides, P. (2017). Software Architecture of the Da Vinci Research Kit. Proc. of the IEEE International Conference on Robotic Computing (IRC), 180-187. 10.1109/IRC.2017.69

Elek, R., Nagy, T. D., Nagy, D. Á., Garamvölgyi, T., Takács, B. \& Galambos, P., ... Haidegger, T. (2017). Towards surgical subtask automation-Blunt dissection. Proc. Of the IEEE 21 st International Conference on Intelligent Engineering Systems, 253-258.

Elek, R., Nagy, T. D., Nagy, D. Á., Kronreif, G., Rudas, I. J., \& Haidegger, T. (2016). Recent Trends in Automating Robotic Surgery. In A. Szakál (Ed.), Proc. Of the 20th IEEE Jubilee International Conference on Intelligent Engineering Systems (pp. 27-32). Budapest: IEEE.

Fei, B., Ng, W. S., Chauhan, S. \& Kwoh, C. K. (2001). The safety issues of medical robotics. Academic Press.

Forbrigger, C., Lim, A., Onaizah, O., Salmanipour, S., Looi, T., Drake, J., \& Diller, E. D. (2019). Cable-Less, Magnetically Driven Forceps for Minimally Invasive Surgery. IEEE Robotics and Automation Letters, 4(2), 1202-1207. doi:10.1109/ LRA.2019.2894504

Gao, Y., Vedula, S. S., Reiley, C. E., Ahmidi, N., Varadarajan, B. \& Lin, H. C. ... Yuh, D. D. (2014). JHU-ISI gesture and skill assessment working set (JIGSAWS): A surgical activity dataset for human motion modeling. Proc. of the MICCAI Workshop: M2CAI, 3. Retrieved from https://cirl.lcsr.jhu.edu/wp-content/uploads/2015/11/ JIGSAWS.pdf

Garg, A., Sen, S., Kapadia, R., Jen, Y., McKinley, S., Miller, L., \& Goldberg, K. (2016). Tumor localization using automated palpation with Gaussian Process Adaptive Sampling. Proc. of the 2016 IEEE International Conference on Automation Science and Engineering (CASE), 194-200. 10.1109/COASE.2016.7743380

Haidegger, T. (2019). Autonomy for Surgical Robots: Concepts and Paradigms. IEEE Trans. on Medical Robotics and Bionics, 1(2), 65-76. doi:10.1109/ TMRB.2019.2913282 
Haidegger, T. (2019). Probabilistic Method to Improve the Accuracy of ComputerIntegrated Surgical Systems. Acta Polytechnica Hungarica. Special Issue on Platforms for Medical Robotics Research, 16(8). doi:10.12700/APH.16.8.2019.8.8

Hannaford, B., Rosen, J., Friedman, D. W., King, H., Roan, P., Cheng, L., ... White, L. (2013). Raven-II: An Open Platform for Surgical Robotics Research. IEEE Transactions on Biomedical Engineering, 60(4), 954-959. doi:10.1109/ TBME.2012.2228858 PMID:23204264

History - Applied Dexterity. (n.d.). Retrieved April 30, 2019, from http:// applieddexterity.com/about/history/

Huang, H.-M. (2008). Autonomy Levels for Unmanned Systems (ALFUS). Academic Press.

Kaber, D. B., \& Endsley, M. R. (2004). The effects of level of automation and adaptive automation on human performance, situation awareness and workload in a dynamic control task. Theoretical Issues in Ergonomics Science, 5(2), 113-153. doi:10.1080/1463922021000054335

Kaplan, K. E., Nichols, K. A., \& Okamura, A. M. (2016). Toward human-robot collaboration in surgery: Performance assessment of human and robotic agents in an inclusion segmentation task. 2016 IEEE International Conference on Robotics and Automation (ICRA), 723-729. 10.1109/ICRA.2016.7487199

Karoly, A. I., Kuti, J. \& Galambos, P. (2018). Unsupervised real-time classification of cycle stages in collaborative robot applications. 2018 IEEE 16th World Symposium on Applied Machine Intelligence and Informatics (SAMI). 10.1109/SAMI.2018.8323994

Kazanzides, P. (2005). Open Source Software Libraries for Computer Integrated Surgery. Retrieved from https://cisst.org/wp-content/uploads/2016/03/YR_8_Open_ Source_Software.pdf

Kazanzides, P., Chen, Z., Deguet, A., Fischer, G. S., Taylor, R. H., \& DiMaio, S. P. (2014). An open-source research kit for the da Vinci® Surgical System. Proc. of the IEEE International Conference on Robotics and Automation, 6434-6439. 10.1109/ICRA.2014.6907809

Lasso, A., Heffter, T., Rankin, A., Pinter, C., Ungi, T., \& Fichtinger, G. (2014). PLUS: Open-Source Toolkit for Ultrasound-Guided Intervention Systems. IEEE Transactions on Biomedical Engineering, 61(10), 2527-2537. doi:10.1109/ TBME.2014.2322864 PMID:24833412 
Li, J., Esteban-Fernández de Ávila, B., Gao, W., Zhang, L. \& Wang, J. (2017). Micro/nanorobots for biomedicine: Delivery, surgery, sensing, and detoxification. Science Robotics, 2(4). doi:10.1126cirobotics.aam6431

Ma, X., Song, C., Chiu, P. W., \& Li, Z. (2019). Autonomous Flexible Endoscope for Minimally Invasive Surgery With Enhanced Safety. IEEE Robotics and Automation Letters, 4(3), 2607-2613. doi:10.1109/LRA.2019.2895273

MacKenzie, L., Ibbotson, J. A., Cao, C. G. L., \& Lomax, A. J. (2001). Hierarchical decomposition of laparoscopic surgery: A human factors approach to investigating the operating room environment. Minimally Invasive Therapy \& Allied Technologies, 10(3), 121-127. doi:10.1080/136457001753192222 PMID:16754003

Márton, L., Szántó,Z., Haidegger, T., Galambos, P., \& Kövecses, J. (2017). InternetBased Bilateral Teleoperation Using a Revised Time—Domain Passivity Controller. Acta Polytechnica Hungarica, 27-45.

McKinley, S., Garg, A., Sen, S., Gealy, D. V., McKinley, J., Jen, Y. \& Goldberg, K. (2016). Autonomous Multilateral Surgical Tumor Resection with Interchangeable Instrument Mounts and Fluid Injection Device. Academic Press.

Mohamed, A., Hossny, M., Nahavandi, S., Dalvand, M., \& Asadi, H. (2018). Towards Trusted Autonomous Surgical Robots. Proc. of the IEEE International Conference on Systems, Man, and Cybernetics (SMC), 4083-4088.

Murali, A., Sen, S., Kehoe, B., Garg, A., McFarland, S., \& Patil, S., ... Goldberg, K. (2015). Learning by observation for surgical subtasks: Multilateral cutting of 3D viscoelastic and 2D Orthotropic Tissue Phantoms. Proc. of the IEEE International Conference on Robotics and Automation, 1202-1209. 10.1109/ICRA.2015.7139344

Nagy, D. Á., Nagy, T. D., Elek, R., Rudas, I. J., \& Haidegger, T. (2018). OntologyBased Surgical Subtask Automation, Automating Blunt Dissection. Journal of Medical Robotics Research, 3(3). doi:10.1142/S2424905X18410052

Nagy, D. Á., Rudas, I. J. \& Haidegger, T. (2018). OntoFlow, a software tool for surgical workflow recording. 2018IEEE 16th World Symposium on Applied Machine Intelligence and Informatics (SAMI), 119-124. 10.1109/SAMI.2018.8323998

Nagy, Tamas D. \& Haidegger, T. (2019). A DVRK-based Framework for Surgical Subtask Automation. Acta Polytechnica Hungarica. Special Issue on Platforms for Medical Robotics Research, 16(8). doi:10.12700/APH.16.8.2019.8.5 
Nagy, T. D., Takács, M., Rudas, I. J., \& Haidegger, T. (2018). Surgical Subtask Automation-Soft Tissue Retraction. Proc. of the 16th IEEE World Symposium on Applied Machine Intelligence and Informatics, 55-60.

Nagyné Elek, R., \& Haidegger, T. (2019). Robot-Assisted Minimally Invasive Surgical Skill Assessment-Manual and Automated Platforms. Acta Polytechnica Hungarica. Special Issue on Platforms for Medical Robotics Research, 16(8). doi:10.12700/APH.16.8.2019.8.9

Nichols, K. A., \& Okamura, A. M. (2013). Autonomous robotic palpation: Machine learning techniques to identify hard inclusions in soft tissues. Proc. of the 2013 IEEE International Conference on Robotics and Automation, 4384-4389. 10.1109/ ICRA.2013.6631198

Olszewska, J. I., Houghtaling, M., Gonçalves, P., Haidegger, T., Fabiano, N., Carbonera, J. L., ... Prestes, E. (2018). Robotic Ontological Standard Development Life Cycle. Presented at the IEEE ICRA 2018 WELCARO workshop. Retrieved from http://real.mtak.hu/86419/

Richter, F., Orosco, R. K. \& Yip, M. C. (2019). Open-SourcedReinforcement Learning Environments for Surgical Robotics. Retrieved from http://arxiv.org/abs/1903.02090

Rosen, J. (2013). Surgical Robotics. In Medical Devices (pp. 63-97). Surgical and Imaging-Guided Technologies.

Seita, D., Krishnan, S., Fox, R., McKinley, S., Canny, J. \& Goldberg, K. (2017). Fast and Reliable Autonomous Surgical Debridement with Cable-Driven Robots Using a Two-Phase Calibration Procedure. Proc. of the 2018 IEEE International Conference on Robotics and Automation (ICRA), 6651-6658. Retrieved from http:// arxiv.org/abs/1709.06668

Sen, S., Garg, A., Gealy, D. V., McKinley, S., Jen, Y., \& Goldberg, K. (2016). Automating multi-throw multilateral surgical suturing with a mechanical needle guide and sequential convex optimization. Proc. of the IEEE International Conference on Robotics and Automation, 4178-4185. 10.1109/ICRA.2016.7487611

Shademan, A., Decker, R. S., Opfermann, J., Leonard, S., Krieger, A., \& Kim, P. C. W. (2016). Supervised autonomous robotic soft tissue surgery. Science Translational Medicine, 8(337), 337ra64. doi:10.1126citranslmed.aad9398 PMID:27147588

Takács, Á., Drexler, D. A., Galambos, P., Rudas, I. J., \& Haidegger, T. (2018). Assessment and Standardization of Autonomous Vehicles. Proc. of the 2018 IEEE 22nd International Conference on Intelligent Engineering Systems (INES), 185-192. 10.1109/INES.2018.8523899 
Takács, Á., Nagy, D. Á., Rudas, I. J., \& Haidegger, T. (2016). Origins of Surgical Robotics: From Space to the Operating Room. Acta Polytechnica Hungarica, 13(1), 13-30.

Takacs, A., Rudas, I., Bosl, D., \& Haidegger, T. (2018). Highly Automated Vehicles and Self-Driving Cars [Industry Tutorial]. IEEE Robotics \& Automation Magazine, 25(4), 106-112. doi:10.1109/MRA.2018.2874301

Vedula, S. S., Malpani, A. O., Tao, L., Chen, G., Gao, Y., Poddar, P., ... Chen, C. C. (2016). Analysis of the Structure of Surgical Activity for a Suturing and Knot-Tying Task. PLoS One, 11(3), e0149174. doi:10.1371/journal.pone.0149174 PMID:26950551

Wei, G. Q., Arbter, K., \& Hirzinger, G. (1997). Real-time visual servoing for laparoscopic surgery. Controlling robot motion with color image segmentation. IEEE Engineering in Medicine and Biology Magazine, 16(1), 40-45. doi:10.1109/51.566151 PMID:9058581

Yamamoto, T., Vagvolgyi, B., Balaji, K., Whitcomb, L. L., \& Okamura, A. M. (2009). Tissue property estimation and graphical display for teleoperated robotassisted surgery. Proc. of the 2009 IEEE International Conference on Robotics and Automation (ICRA), 4239-4245. 10.1109/ROBOT.2009.5152674

Yang, G.-Z., Cambias, J., Cleary, K., Daimler, E., Drake, J., Dupont, P. E., ... Taylor, R. H. (2017). Medical robotics-Regulatory, ethical, and legal considerations for increasing levels of autonomy. Science Robotics, 2(4). doi:10.1126cirobotics.aam8638

\section{ENDNOTES}

1 https://ec.europa.eu/digital-single-market/en/news/attitudes-towards-impactdigitisation-and-automation-daily-life

2 https://transmitter.ieee.org/ai/ 